RESEARCH ARTICLE

\title{
Sevoflurane but not Propofol Induces Immunomodulatory Effects in Patients Undergoing Aortic Valve Replacement and Cardiopulmonary Bypass
}

\section{Sonia Veiras ${ }^{1 *}$, Raquel Rodríguez González ${ }^{1,2}$, Tomás Sobrino ${ }^{3}$, Javier Rodríguez ${ }^{4,5}$, Aurora Baluja ${ }^{1}$ and Julián Álvarez ${ }^{1}$}

${ }^{1}$ Critical Patient Translational Research Group, Department of Anesthesiology, Intensive Care and Pain Management, Hospital Clínico Universitario, Instituto de Investigación Sanitaria (IDIS), University of Santiago de Compostela, Santiago de Compostela, Spain

${ }^{2}$ Department of Pathology, University of Santiago Faculty of Nursery, Santiago de Compostela, Spain

${ }^{3}$ Department of Neurology, Neurovascular Area, Clinical Neurosciences Research Laboratory, Hospital Clínico Universitario, Health Research Institute of Santiago de Compostela (IDIS), University of Santiago de Compostela, Santiago de Compostela, Spain

${ }^{4}$ Department of Biochemistry and Molecular Biology, University of Santiago de Compostela, Santiago de Compostela, Spain ${ }^{5}$ Department of Biochemistry, Hospital Clínico Universitario, Health Research Institute of Santiago de Compostela (IDIS), Santiago de Compostela, Spain

* Corresponding author: Sonia Veiras Del Río, Critical Patient Translational Research Group, Department of Anesthesiology, Intensive Care and Pain Management, Hospital Clínico Universitario, Instituto de Investigación Sanitaria (IDIS), University of Santiago de Compostela, Santiago de Compostela, Spain, Tel: +34-981-950-674, Fax: +34-981-950-634, E-mail: veiras1@gmail.com

\begin{abstract}
Background: Volatile anaesthetics, most of all sevoflurane, have been described as providers of myocardial preconditioning, but few articles are focused on immunomodulatory effects of these agents. We aimed to study the effects of different anaesthetic procotols with sevoflurane and propofol on immunomodulation in patients undergoing cardiopulmonary bypass (CBP).

Methods: Twenty-five patients scheduled for aortic valve replacement undergoing CBP were studied and divided in three groups depending on anaesthetic protocol: sevoflurane for induction, maintenance and CBP period (group 1); propofol for induction, maintenance and CBP period (group 2); propofol for induction and CBP period and sevoflurane for maintenance before and after CBP (group 3). Blood samples were obtained at baseline, immediately after sternal closure, 24 hours, 72 hours and 7 days after surgery. TLR2 and TLR4 expression were measured in monocytes and lymphocytes, and serum levels of tumoral necrosis factor a (TNFa), Cystatin C, Reactive C Protein (PCR), Propeptide Brain Natriuretic Protein (Pro-BNP) and Interleukin (IL) 6, IL-2R and IL-8 were analyzed.
\end{abstract}

Conclusions: Compared with propofol, sevoflurane anaesthesia was associated to lower expression of TLR2 in monocytes and lower serum levels of inflammatory mediators.

\section{Keywords}

Sevoflurane, Propofol, Extracorporeal circulation, Toll-Like receptors, Inflammation, Anaesthesia

\section{Introduction}

Cardiopulmonary bypass or extracorporeal circulation (CBP, ECC) is extensively used worldwide as a supportive method for patient organic functions while cardiac surgery procedures are developed. However, although essential, CBP is not a harmless support and widespread inflammatory response is a well-known aftereffect. This inflammatory reaction occurs in two phases [1]: 1) Early, mediated by coagulation system, complement and fibrinolysis and 2) Late, secondary to ischemia-reperfusion reactions and endotoxins. 
Toll-like receptors (TLRs) are a family of highly conserved innate immunity receptors whose main function is the recognition of nearly any pathogen agent by linking to the so-called "pathogen associated molecular patterns" (PAMPs). This union between TLRs and PAMPs leads to innate immunological response activation, production of inflammatory mediators and phagocytosis and opsonization phaenomena [2]. TLRs are widely spread on cellular types of immune system, but after investigating a wider role for TLRs, it was discovered that non-pathogen stimuli (i.e. mechanical damage, necrosis) are also able to trigger an immune response as they are recognized by TLRs. These non-pathogen molecules have been named DAMPs (Damage Associated Molecular Pattern) [3].

Much has been written about sevoflurane cardio protection in terms of pre [4] and postconditioning [5] but progressively we can find in scientific papers new characteristics of this anaesthetic gas beyond properly hypnotic effects.

Our research group has recently described that exposure to sevoflurane can influence TLR cellular expression and therefore inflammatory response [6], so we hypothesized about an immunomodulatory role for sevoflurane anesthesia in patients undergoing cardiopulmonary bypass for cardiac procedures.

\section{Methods}

\section{Study design}

We conducted a prospective observational trial, including patients diagnosed of severe aortic valve stenosis and scheduled for aortic valve replacement under ECC in our university hospital. We selected this population because its clinical and demographic features are more homogeneous than in coronary artery disease patients scheduled for myocardial revascularization. No randomization was applied to patient's inclusion because our aim was to compare TLRs expression and inflammatory mediators in patients under different anaesthetic protocols routinely used in day case surgery depending on anaesthesiologist preferences, so no specific restrictions or interventions were established.

Patients were excluded in case of coronary artery bypass graft needed in the same surgical act than aortic valve replacement, as well as emergencies, endocarditis and chronic steroid therapies.

This research was carried out in accordance with the Declaration of Helsinki of the World Medical Association (2008) and approved by the Ethics Committee of the Servizo Galego de Saúde (2011/409). Informed consent was obtained from each patient or their relatives after full explanation of the procedures.

\section{Surgical technique}

Aortic valve replacement is performed under ster- notomy, pericardial dissection and heart cannulation (aorta and right atrium) to provide an extracorporeal circuit for extracorporeal circulation. Total systemic heparinization (300 $\mathrm{Ul}$ per $\mathrm{kg}$ ) is essential to avoid thrombotic events during pump period.

Sick aortic valve is removed through an aortotomy and a prosthetic valve (mechanical or biological depending on patient profile) is sutured to the aortic annulus.

After aortotomy closure, extracorporeal circulation is interrupted and heart beating, and pulmonary ventilation is restarted.

After heart decannulation and heparin reversion with protamine, sternotomy is closed, and the patient is transferred to a Critical Postoperative Unit.

\section{Anaesthetic protocols}

The patients were assessed preoperatively on the day before surgery and informed consent was obtained.

As a premedication was administered sublingual alprazolam $0.25 \mathrm{mg}$ one hour before the transfer of the patient to the operating room.

The anaesthetic preparation included connection of patient to the monitor (General Electric, Advance ${ }^{\circledast}$ ), placement of peripheral venous access 14 G, 20 G arterial line in the radial or brachial artery and sensor placement BIS (BIS/XP, Aspect Medical Systems, USA). Anaesthesiologist in charge of each case applied his/her habitual technique. Three different protocols were identified: 1 ) Total inhalatory anaesthesia with sevoflurane for induction, maintenance and ECC period, 2) Total intravenous anaesthesia with propofol for induction, maintenance and ECC period and 3) Mixed anaesthetic protocol using propofol for induction and ECC period and sevoflurane for maintenance between induction and ECC.

Rocuronium (Braun ${ }^{\circledR}, 10 \mathrm{mg} / \mathrm{ml}$, bolus for tracheal intubation of $0.1 \mathrm{mg} / \mathrm{kg}$ ) was the most employed muscle relaxant agent and fentanyl (Fentanest ${ }^{\circledR}$ Kern Phar$\mathrm{ma}$, boluses of 3 microgrs per $\mathrm{kg}$ ) for analgesic purposes was liberally administered.

The laryngoscopy and tracheal intubation were made following administration of muscle relaxant agent (Rocuronium) and the patient was connected to the anaesthesia machine (General Electric, Advance ${ }^{\circledR}$ ) appliying tidal volumes of $6 \mathrm{ml}$ per $\mathrm{kg}$, positive end expiratory pressure of at least $6 \mathrm{~cm} \mathrm{H}_{2} \mathrm{O}$ and respiratory rate of 1012 breaths per minute. The maintenance of anaesthesia was obtained with propofol (Diprivan) in dosage 5-6 $\mathrm{mg} / \mathrm{kg} / \mathrm{h}$, with continuous infusion pumps (Fresenius, Vial, Brezins, France) or by sevoflurane continuous inhalation to get MAC 1-1.5. In both cases the goal was to obtain BIS values between 45 to 55 . In patients the right jugular vein was catheterized and trilumen catheter and sheath 8.5 French were placed.

Patients were under continuously temperature con- 
trol with special catheter bladder and a second measure obtained through nasopharyngeal probe.

Blood gases, the acid-base balance, blood sugar and serum electrolytes and lactate concentration were monitored periodically. Adequacy of haemostasis was checked periodically with time Activated Clotting Time (ACT) with a goal value of $>400$ seconds for a safety anticoagulation on cannulation maneuvers and on CBP.

Twenty minutes after reversal with protamine, both ACT and thromboelastogram $\left(\right.$ ROTEM $\left.^{\circledR}\right)$ were performed to correct, if necessary, any aspect of haemostasis by a goal-directed technique.

After surgery, patients were transported to Postoperative Care Unit for close monitoring. Fast trach protocol was applied so to get patients extubated during the first six hours of postoperative period if clinical and gasometric parameters were favorable.

\section{Sample collection}

In order to analyze a temporal profile, blood samples (S) were collected at different time points: Basal sample, before anaesthetic induction (S1), after ECC, during wound closure (S2); 24 hours after S1 (S3); 72 hours after S1 (S4); at discharge, 5-7 days after surgery (S5). At each time point, one EDTA-anticoagulated tube and one biochemistry tube were withdrawn for TLRs expression and biomarkers analysis, respectively. Biochemistry tubes were centrifuged at $3000 \times \mathrm{g}$ for 10 minutes, and serum was immediately frozen and stored at $-80^{\circ} \mathrm{C}$ until analysis.

\section{Molecular determinations}

Toll-like receptors 2 and 4 expression analysis: Toll-like receptors 2 and 4 expression analyses were performed by flow cytometry [7] in blood samples. For the expression analysis of TLR2 and TLR4, monocytes, lymphocytes, and neutrophils were separated by their forward and side scattering signal characteristics on flow cytometry. APC-TLR2 antibody (BD Biosciences, NJ, USA) and biotin-TLR4 antibody (BD Biosciences) together with streptavidin-PE (BD Biosciences) were used to quantify TLR expression. Samples were analyzed on a FACSAria flow cytometer (BD Biosciences) using FACSDiva 6.0 software. Mean expression of TLR2 and TLR4 on monocytes was analysed and expressed as AFU (arbitrary fluorescence units).

Biomarkers analysis: Biomarkers were measured in serum samples by investigators blinded to clinical data using immunoassay-based chemiluminescent automated systems: Immulite ${ }^{\circledR}$ (Siemens Healthcare, Madrid, Spain) for tumoral necrosis factor $\alpha(T N F \alpha)$, C-reactive protein (CRP), and Interleukin (IL) 6, IL-8, IL-1 $\beta$ and IL$2 \mathrm{R}, \mathrm{N}$ latex kit for Cystatin C (Siemens Healthcare, Madrid, Spain) and Elecsys ${ }^{\circledR} 2010$ (Roche, Madrid, Spain) for pro-brain natriuretic peptide (Pro-BNP). Inter- and intra assay coefficients of variation were below $5 \%$ for all molecules.

\section{Statistical analysis}

The results were expressed as percentages for categorical variables and as mean (SD) or median [quartiles] for the continuous variables depending on their normal distribution or not, respectively. The KolmogorovSmirnov test was used for testing the normality of the distribution. All variables showed a normal distribution, except body mass index (BMI). Proportions were compared using the $\chi^{2}$ test, while the continuous variables between groups were compared with the Student's $t$ (variables with normal distribution) or U-Mann Whitney (variables with non-normal distribution) tests. Bivariate correlations were studied with Pearson analysis. Statistical differences were considered significant when $p<$ 0.05 . Results are expressed as adjusted odds ratio (OR) with 95\% confidence intervals ( $\mathrm{Cl}$ 95\%). Statistical analysis was made using SPSS 19.0 for Mac.

\section{Results}

We enrolled 25 patients for this study.

Patients were classified in three groups:

- Group 1 (10 patients): Sevoflurane anaesthesia

- Group 2 (6 patients): Propofol anaesthesia

- Group 3 (9 patients): Mixed propofol-sevoflurane anaesthesia

Table 1 reports the baseline characteristics of the patients by allocation.

All patients were white.

We established four univariate studies:

- Sevoflurane versus propofol during the whole anaesthetic time (including CBP): In patients anesthetized only with propofol, a higher TLR2 expression was evidenced in monocytes immediately after surgery and at 24 and 72 postoperative hours. IL6 presented higher plasma values at 24 postoperative hours and also in $7^{\text {th }}$ postoperative day. TNF $\alpha$ after sternal closure was also higher in propofol group. ProBNP and IL2R showed higher values on day seven after surgery in propofol anesthesia.

- Sevoflurane versus propofol for maintenance period (before and after CBP): Patients who received anaesthetic maintenance with propofol showed stronger expression of TLR2 in monocytes at 72 hours after surgery and higher TNF $\alpha$ inmediately after surgery. ProBNP and IL2R were higher in those patients maintained with propofol than in those maintained with sevoflurane.

- Sevoflurane only versus mixed anaesthesia (propofol-sevoflurane): Patients who received mixed anaesthetic scheme showed more expression of TLR2 in monocytes inmediately after surgery and at 24 and 72 postoperative hours. TNF $\alpha$ after surgery and IL6 at 24 postoperative hours were also higher in mixed anaesthesia group. Contrary to our hypothesis, in those patients anaesthesized 
Table 1: Baseline characteristics of our study population.

\begin{tabular}{|c|c|}
\hline Age $( \pm S D)$ & $73.0 \pm 9.7$ years \\
\hline Men, n (\%) & $14(56.0)$ \\
\hline Arterial Hypertension, $\mathrm{n}(\%)$ & $18(72.0)$ \\
\hline Diabetes, n (\%) & $6(24.0)$ \\
\hline Atrial fibrillation, $\mathrm{n}(\%)$ & $6(24.0)$ \\
\hline Betablocker therapy, $\mathrm{n}(\%)$ & $6(24.0)$ \\
\hline Diuretics therapy, n (\%) & $11(44.0)$ \\
\hline Antiplatelet therapy, $\mathrm{n}(\%)$ & $6(24.0)$ \\
\hline Statins therapy, n (\%) & $12(48.0)$ \\
\hline Oral antidiabetic therapy, $\mathrm{n}(\%)$ & $6(24.0)$ \\
\hline BMI, median (percentil 25, percentil 75) & $29(27,32)$ \\
\hline \multicolumn{2}{|l|}{ NYHA, n (\%) } \\
\hline Class I, n (\%) & $2(8.0)$ \\
\hline Class II, n (\%) & $21(84.0)$ \\
\hline Class III, n (\%) & $2(8.0)$ \\
\hline Preserved myocardial function, $\mathrm{n}(\%)$ & $23(92.0)$ \\
\hline Total surgical time (min) & $192.2 \pm 64.2$ \\
\hline ECC time (min) & $82.9 \pm 21.0$ \\
\hline Aortic clamp time (min) & $70.5 \pm 16.0$ \\
\hline $\begin{array}{l}\text { Mechanical ventilation time after surgery } \\
\text { (hours) }\end{array}$ & $7.3 \pm 5.7$ \\
\hline Endotracheal intubation time (hours) & $10.0 \pm 6.7$ \\
\hline $\begin{array}{l}\text { Length of stay in Postoperative Care Unit } \\
\text { (hours) }\end{array}$ & $52.0 \pm 57.0$ \\
\hline Length of stay at hospital after surgery (days) & $6.0 \pm 2.7$ \\
\hline Peak of Troponin I I (ng/dL) & $6.4 \pm 3.6$ \\
\hline Leucocyte peak, $\times 10^{3} / \mathrm{ml}$ & $9.3 \pm 3.6$ \\
\hline Electric defibrillation required after CBP $\mathrm{n}(\%)$ & $5(20.0)$ \\
\hline Biologic Prosthesis, n (\%) & $16(64.0)$ \\
\hline Low cardiac output after surgery, n (\%) & $4(16.0)$ \\
\hline Neurologic impairment, n (\%) & $2(8.0)$ \\
\hline Complications after discharge, $\mathrm{n}(\%)$ & $4(16.0)$ \\
\hline Platelet transfusion, $\mathrm{n}(\%)$ & $7(28.0)$ \\
\hline Fresh frozen plasma transfusion, $\mathrm{n}(\%)$ & $6(24.0)$ \\
\hline \multicolumn{2}{|l|}{ Red cell transfusion $(n=25)$ : } \\
\hline $0, \mathrm{n}(\%)$ & $6(24.0)$ \\
\hline $1-3, n(\%)$ & $12(48.0)$ \\
\hline$>3, \mathrm{n}(\%)$ & $7(28.0)$ \\
\hline \multicolumn{2}{|l|}{ Anesthetic agent for induction $(n=25)$ : } \\
\hline Sevoflurane, n (\%) & $10(40.0)$ \\
\hline Propofol, n (\%) & $10(40.0)$ \\
\hline Etomidate, n (\%) & $5(20.0)$ \\
\hline \multicolumn{2}{|l|}{ Anesthetic agent for maintenance $(n=25)$ : } \\
\hline Sevoflurane, n (\%) & $19(76.0)$ \\
\hline Propofol, n (\%) & $6(24.0)$ \\
\hline \multicolumn{2}{|l|}{ Anesthetic agent during CBP $(n=25)$ : } \\
\hline Sevoflurane, n (\%) & $10(40.0)$ \\
\hline Propofol, n (\%) & $14(56.0)$ \\
\hline Midazolam, n (\%) & $1(4.0)$ \\
\hline
\end{tabular}

only with sevoflurane, IL8 was higher on samples obtained 72 hours and seven days after surgery.

$\odot$ Propofol only versus mixed anaesthesia (propofol-sevoflurane): Both groups have similar levels in TLR2 monocyte expression at any of the samples obtained, but higher levels of proBNP on day seven and of IL8 at 72 hours after surgery were observed in those patients under "propofol-only" anaesthesia.
Table 2: Synthesis of our results, showing only those with statistically significant values.

Four comparative studies showed higher level of TLRs and inflammatory mediators in blood samples obtained from patients who received only propofol or mixed anaesthesia with propofol and sevoflurane. The only exception was IL8 at 72 hours and at 7 days after surgery when compared sevoflurane only versus mixed anaesthesia. IL8 was higher in sevoflurane anaesthesia group.

Statistically signification achieved $(p<0.05)$

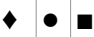

TLR2 monocytes immediately

after surgery

TLR2 monocytes 24 hours

TLR2 monocytes 72 hours

IL-6 24 hours

IL-6 day 7 after surgery

TNFa immediately after surgery +++

proBNP day 7 after surgery

IL-2R day 7 after surgery

$++$

$++$

$++$

$++$

$+-$

$++-$

$++$

IL-8 72 hours after surgery _- - + (> In sevoflurane) +

IL-8 day 7 after surgery $\quad-\quad++(>$ In sevoflurane) -

-Sevoflurane versus propofol during the whole anaesthetic time (including CBP); • Sevoflurane versus propofol for maintenance period (before and after CBP); - Sevoflurane only versus mixed anaesthesia (propofol-sevoflurane); $\odot$ Propofol only versus mixed anaesthesia (propofol-sevoflurane).

Table 2 presents a synthesis of our results showing only those with statistically significant values.

We did not find any differences in TLR4 expression between the different anaesthetic groups, nor in monocytes neither in lymphocytes.

Significant differences were found in TLR2 expression but only in monocytes, not in lymphocytes.

Regarding plasmatic inflammatory mediators, IL1 $\beta$ was measured in all the blood samples but it was excluded of the statistical analysis because its plasma levels was $<5$ Units per $\mathrm{ml}$ without changes throughout the temporal profile. Recent publications [8] have evidenced a relation between IL1 $\beta$ and inflammatory mechanisms inducing aortic stenosis, so it seemed interesting for us its determination in our profile but could not find significant variations.

\section{Discussion}

In this study, we have found that anaesthetic regimen can influence inflammatory response since its molecular basis on Toll Like receptors expression.

Extracorporeal circulation induces an inflammatory response that is quite well known in terms of clinical and biochemical manifestations but is rather unknown with regard to its molecular and cellular genesis.

By demonstrating changes in TLR expression and its relationship with anaesthetic regimen, we have tried to glimpse new aspects for our daily practice beyond purely anaesthetic ones.

Maybe we are approaching innovative expectatives and maybe we (anaesthesiologists) should start to consider our intervention on patients under surgical proce- 

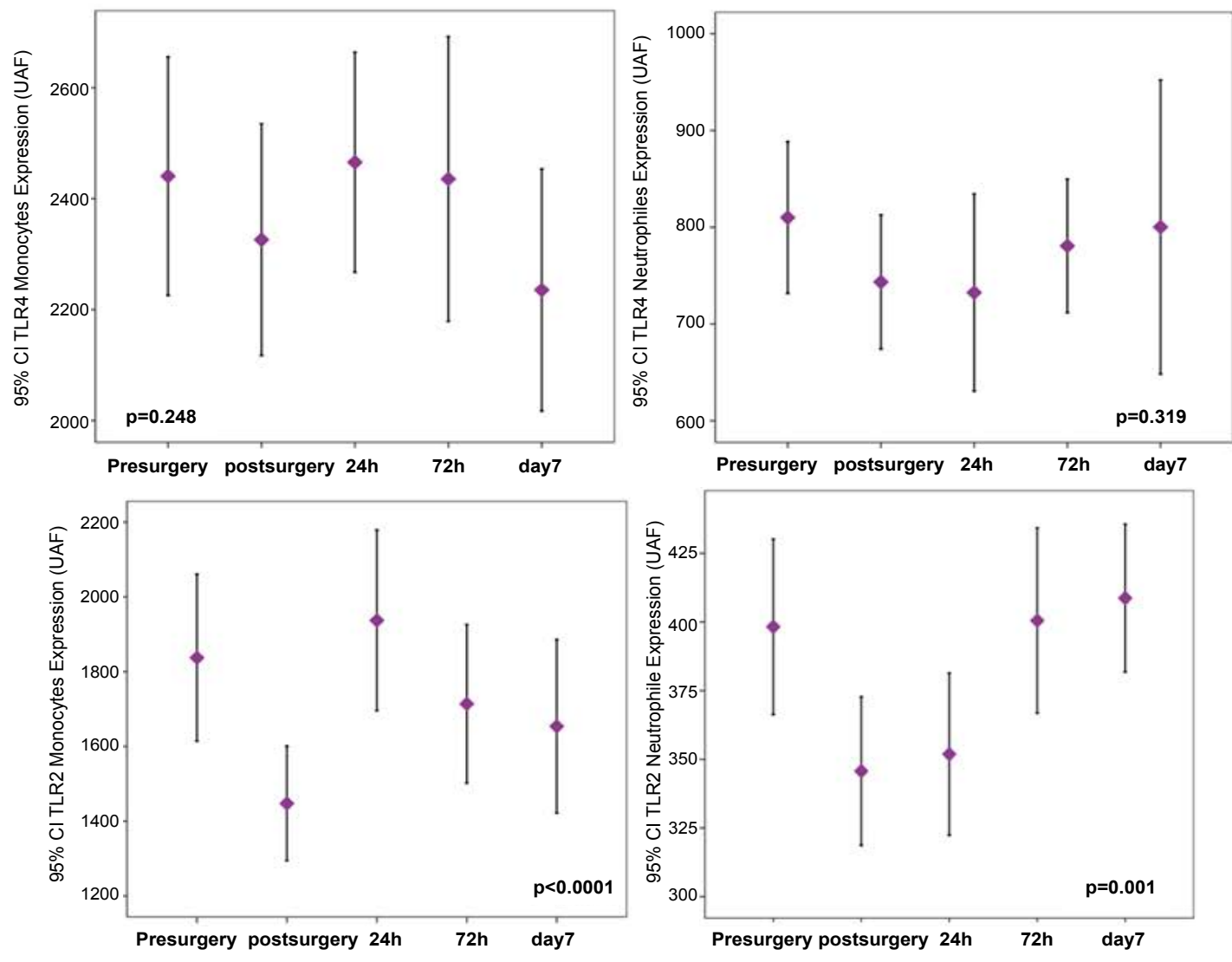

Figure 1: Temporary evolution of TLR2 and TLR4 in monocytes and in neutrophyles in our population study.
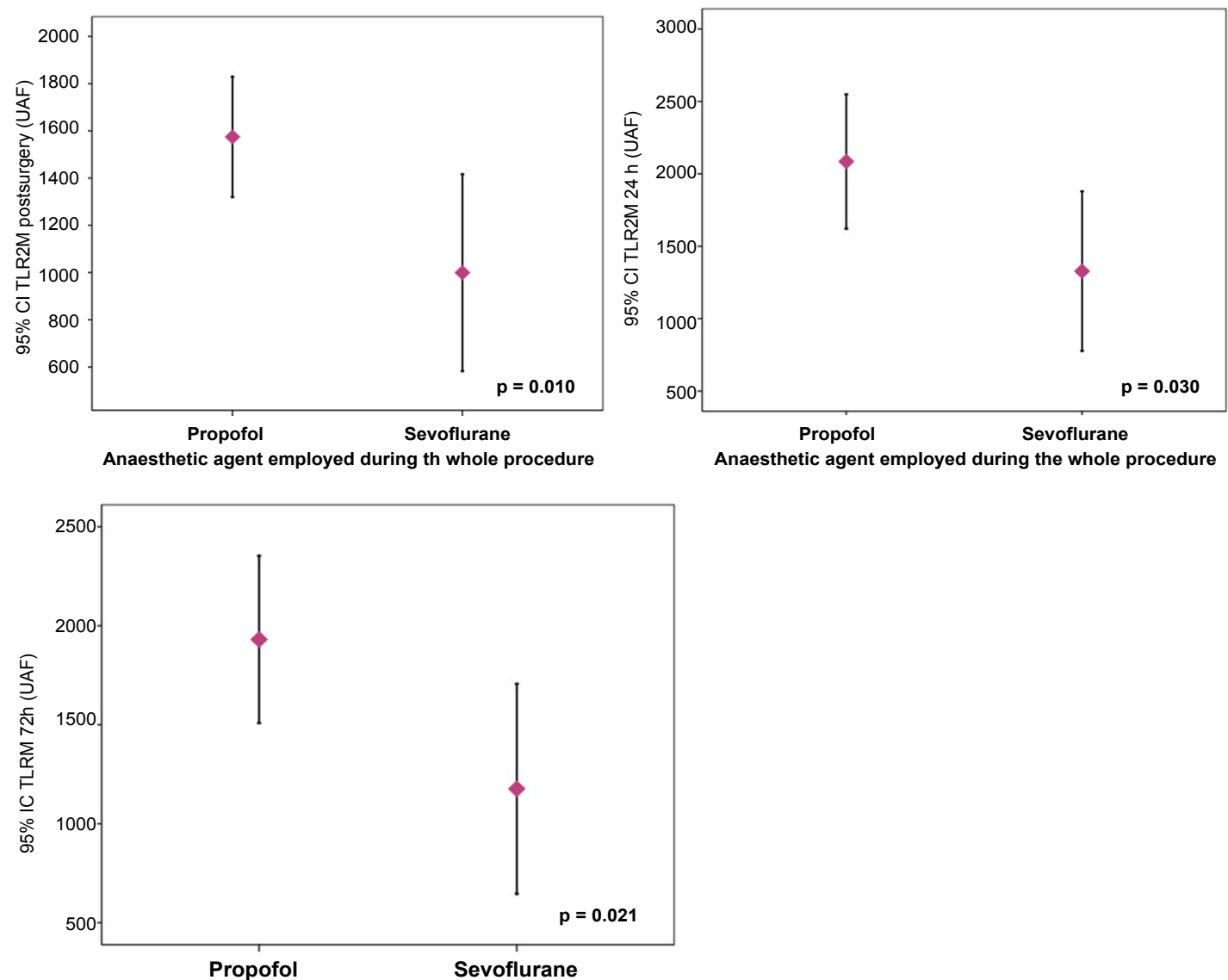

Anaesthetic agent employed during the whole procedure

Figure 2: Differences in TLR2 expression when compared propofol anaesthesia with sevoflurane anaesthesia.

dures not only like a necessary evil but like an essential part of patient's therapy.
Temporary profile in our population regarding TLR modifications after ECC with independence of anaes- 
thetic protocol is concordant with a previous publication [9] that showed reductions in TLR2 and TLR4 in monocytes and in neutrophyles inmediately after ECC, with increases up to $120 \%$ in TLR2 and TLR4 expression 24 hours after ECC.

(Figure 1 shows the profile of TLRs expression obtained in our study).

When we made the comparison between both "pure" anaesthetic groups (sevoflurane only versus propofol only), we noted significant differences in temporal TLR2 expression in monocytes, as illustrated in Figure 2.

(Figure 2: differences in TLR2 expression when compared propofol anaesthesia with sevoflurane anaesthesia).

\section{Strengths and limitations}

We are aware of the following limitations. First, we did not use randomization protocol, so patients enrollment was enlarged until we achieved a comparable number of patients per anaesthetic group; and second, a larger number of patients is necessary to establish differences in clinical outcomes between patients with several degrees of inflammatory response.

\section{Conclusions}

Sevoflurane anaesthesia induces immunomodulatory effects on patients scheduled for aortic valve replacement, with less expression of TLRs and lower release of inflammatory mediators.

If our results should be confirmed in a larger series of patients, maybe we should raise that pure propofol anaesthesia is not the most appropriate for patients scheduled for surgery under CBP, at least in regard to inflammatory response.

\section{Disclosures}

The authors manifest not having any conflict of interests.

-Sonia María Veiras del Río is anaesthesiologist in Santiago de Compostela. She has written her doctoral thesis on cardiopulmonary bypass and immunomodulatory effects of several anaesthetic drugs. She drives Cardiovascular Anaesthesia Section at University Hospital of Santiago de Compostela.

-Raquel González is doctor in Biology and she has directed several investigations in critical care patients.
She is professor at Nursery Faculty in Santiago de Compostela.

-Tomás Sobrino is doctor in Biology and he is driving numerous research studies on cerebrovascular diseases. He has quantified TLR in plasma samples and has supervised statistical work.

-Javier Rodríguez belongs to Biochemistry and Molecular Medicine Department at University Hospital in Santiago de Compostela, and he has supervised all the results of inflammatory mediators.

-Aurora Baluja is anaesthesiologist in Santiago de Compostela and she is a fundamental part of the Translational Research Group.

-Julián Alvarez is head of Anaesthesia Department and Cathedratic of Anaesthesia in the University of Santiago de Compostela.

\section{References}

1. Laffey JG, Boyland JF, Cheng DC (2002) The systemic inflammatory response to cardiac surgery : Implications for the anesthesiologist. Anesthesiology 97: 215-222.

2. Kawai T, Akira S (2009) The roles of TLRs, RLRs and NLRs in pathogen recognition. Int Immunol 21: 317-337.

3. Li Yu, Wang I, Cheng S (2010) Endogenous toll-like receptor ligands and their biological significance. $\mathrm{J}$ Cell Mol Med 14: 2592-2603.

4. Landoni G, Fochi O, Tritapepe L, Guarracino F, Belloni I, et al. (2009) Cardiac protection by volatile anesthetics. A review. Minerva Anesthesiol 75: 269-273.

5. Zhang F, Chen G, Chen C, Yan M (2009) Sevoflurane postconditioning converts persistent ventricular fibrillation into regular rhythm. Eur J Anaesthesiol 26: 766-771.

6. Rodríguez González R, Baluja A, Veiras del Río S, Rodríguez A, Rodríguez J, et al. (2013) Effects of sevoflurane postconditioning on cell death, inflammation and TLR expression in human endotelial cells exposed to LPS. J Transl Med 11: 87.

7. Brea D, Blanco M, Ramos Cabrer P, Moldes O, Arias S, et al. (2011) Toll-like receptors 2 and 4 in ischemic stroke: Outcome and therapeutic values. J Cereb Blood Flow Metab 31: 1424-1431.

8. Nadionek N, Lee JH, T Brett Reece, Michael J Weyant, Joseph C Cleveland, et al. (2013) Interleukin-1 beta induces an inflammatory phenotype in human aortic valve interstitial cells through nuclear factor kappa Beta. Ann Thorac Surg 96: 155-162.

9. Hadley J, Wang Jacob, Michaels Louis, Dempsey CM, Foster SJ, et al. (2007) Alterations in inflammatory capacity and TLR expression on monocytes and neutrophils after cardiopulmonary bypass. Shock 27: 466-473. 